\title{
Design of Microstrip Leaky Wave Antenna Composed of Composite Right/Left Handed Transmission Line
}

\author{
Yanchao $\mathrm{Wu}^{1, \mathrm{a}^{*}}$, Yawen $\mathrm{Dai}^{2, \mathrm{~b}}$ Xin Xiong ${ }^{3, \mathrm{c}}$ \\ ${ }^{1}$ WuHan University of Technology, WuHan, 430070, China \\ ${ }^{2}$ WuHan University of Technology, WuHan, 430070, China \\ ${ }^{3}$ WuHan University of Technology, WuHan, 430070, China \\ a287967149@qq.com, bdaiyaweny@163.com, '104721133@qq.com \\ ${ }^{*}$ corresponding author:Tel:+8618171290919
}

Keywords: leaky-wave antenna; composite right/left-handed transmission line; scanning beam; radiation characteristic

\begin{abstract}
In this paper, by referring to the new equivalent model, I designed and made three kinds of antenna objects of composite right/left-handed transmission line with microstrip leaky-wave antenna. By using network analyzer, I measure their $S$ parameter and the result showed that the physical model, circuit model and antenna object all have good consistency. In order to make further study on the characters of space scanning beam on composite right/left-handed transmission line, I simulate the far field radiation characteristics for above three antenna objects and he made the object test in the anechoic chamber. I drew such conclusion: under the equilibrium state, composite right/left-handed transmission line with microstrip leaky-wave antenna has more even radiation characteristic above different beams.
\end{abstract}

\section{Introduction:}

In radar and wireless communication system, array antenna usually realizes the scanning beam, by using the phase shifter or controlling the formed digital beam. But such scanning beam method takes great space with higher cost, so it does not accord with the design philosophy of miniaturization and low $\operatorname{cost}^{[1]}$.

Microstrip leaky wave antenna's main beam can change with the frequency scan, but the traditional microstrip leakage wave antenna can only achieve a single beam scanning, the electromagnetic properties of left-handed metamaterials can make the microstrip leaky wave antenna beam scanning range is extended to the whole space, realize the angle range of radiation for $-90^{\circ}-+90^{\circ}$, and will not affect the antenna radiation efficiency.

\section{Circuit extraction based on resonant frequency}

In the design of multi section CRLH transmission line structure, usually use a single section of the CRLH transmission line structure to extract parameters, but due to the design methods ignore the coupling between each section of the CRLH structure, actually single-cell CRLH transmission line structure simulation to get the transfer matrix does not accurately describe ten Festival CRLH transmission line structure, even larger deviations ${ }^{[2]}$. While extracting the equivalent circuit parameters of the CRLH transmission line structure, we usually used a large frequency range of data to extract the circuit parameters, then the upper limit and the lower cutoff frequency are obtained. This method not only has a large amount of computation, but also the result of the extraction will fluctuate over a range of frequencies, we cannot judge which set of data is the description of the physical structure. New circuit extraction method, starting from the physical characteristics, first to find the upper and lower cut-off frequency, and then to solve the circuit parameters. This method is not only convenient and simple, but also higher accuracy, but also can calculate the circuit loss.

Mainly includes the following steps:

1) Removal of microstrip lines 
In the design process of a leaky wave antenna, in order to eliminate the influence of higher modes on the S parameters, both ends of the CRLH transmission line structure respectively with a microstrip line. But because of the microstrip line, the electromagnetic wave propagation in CRLH transmission line when there is phase delay, in order to accurately extract the parameters of circuit elements, we need to eliminate the influence of the microstrip line first ${ }^{[3]}$.

In the solution of the HFSS model simulation, the influence of the microstrip line can be removed by setting the deembed option. In the modeling process of ADS circuit model, we only considered the model of ten CRLH transmission line. The results of antenna physical S parameters measured by vector network analyzer, we need to remove the two section of the microstrip line of the $\operatorname{matrix}^{[4]}$.

2) Calculation of the ABCD matrix of the CRLH transmission line structure of a single unit

After removing the phase delay of the microstrip line, we can get a new $S$ parameter, this $S$ parameter is the S parameter of the whole CRLH transmission line structure.

According to the microwave network theory, the $S$ parameters of the two port network and the ABCD matrix can be transformed into each other, so the ABCD matrix of the CRLH transmission line can be calculated by the formula.And also according to the theory of microwave network, when a two port network comprises a plurality of cascaded two port networks and ABCD matrix, the ABCD matrix of the two port network can be obtained by multiplying the ABCD matrix of a plurality of two port networks ${ }^{[5]}$.

The CRLH transmission line in this paper is composed of 10 identical transmission lines, so the $\mathrm{ABCD}$ matrix of each structure unit is:

$$
\left[\begin{array}{ll}
A & B \\
C & D
\end{array}\right]_{U C}=\left[\begin{array}{ll}
A & B \\
C & D
\end{array}\right]^{\frac{1}{10}}
$$

$\left[\begin{array}{ll}A & B \\ C & D\end{array}\right]_{U C}$ represents the ABCD matrix of a unit structure CRLH transmission line.

3) $\mathrm{Z}$ matrix expression

After getting the $\mathrm{ABCD}$ matrix of the structure unit of the transmission line, we can calculate the impedance matrix ( $Z$ matrix) of the structure element.

Because of the relationship between the $\mathrm{Z}$ matrix and $\mathrm{ABCD}$ matrix of the transmission line structure in this paper:

$$
\left[\begin{array}{ll}
A & B \\
C & D
\end{array}\right]_{U C}=\left[\begin{array}{ll}
1 & Z \\
0 & 1
\end{array}\right]\left[\begin{array}{cc}
1 & 0 \\
Y & 1
\end{array}\right]=\left[\begin{array}{cc}
1+Z Y & Z \\
Y & 1
\end{array}\right]
$$

Therefore, the expression of the impedance and admittance can be obtained:

$$
\begin{aligned}
& Z=B \\
& Y=C
\end{aligned}
$$

Based on the simulation data of the ten section, the D element in the single node transmission matrix is 1 , the only solution that meets the physical properties can be selected.

4) The establishment of a lossy circuit model

Traditional CRLH equivalent circuit model is shown in Figure1. 


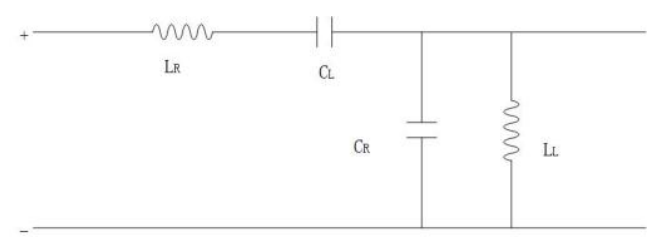

Figure1 Traditional CRLH equivalent circuit model

Improved new CRLH equivalent circuit model is shown in Figure2.

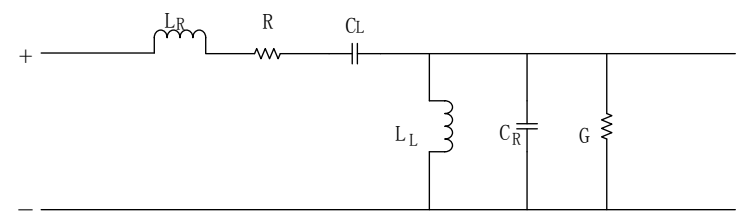

Figure2 Improved new CRLH equivalent circuit model

5) The derivation and calculation of circuit parameters

The influence of the resistance and the conductance of the circuit is considered:

$$
\begin{aligned}
& Z=R+j \omega L_{R}\left(1-\frac{\omega_{s e}{ }^{2}}{\omega^{2}}\right) \\
& Y=G+j \omega C_{R}\left(1-\frac{\omega_{s h}{ }^{2}}{\omega^{2}}\right)
\end{aligned}
$$

It can be known that the expression of resistance and conductance in the circuit is:

$$
\begin{aligned}
& R=\operatorname{Re} Z=\operatorname{Re} B \\
& G=\operatorname{Re} Y=\operatorname{Re} C
\end{aligned}
$$

The derivation of the imaginary part of the equation:

$$
\begin{aligned}
& \frac{\mathrm{d} \operatorname{Im} Z}{\mathrm{~d} \omega}=L_{R}\left(1+\frac{\omega_{s e}{ }^{2}}{\omega^{2}}\right) \\
& \frac{\mathrm{d} \operatorname{Im} Y}{\mathrm{~d} \omega}=C_{R}\left(1+\frac{\omega_{s h}{ }^{2}}{\omega^{2}}\right)
\end{aligned}
$$

When the independent variables are satisfied with $\omega=\omega_{s e}$ and $\omega=\omega_{s h}$, we can quickly get the expression of the right hand inductance and capacitance:

$$
\begin{aligned}
& L_{R}=\frac{1}{2} \operatorname{Im} Z^{\prime}\left(\omega_{s e}\right) \\
& C_{R}=\frac{1}{2} \operatorname{Im} Y^{\prime}\left(\omega_{s h}\right)
\end{aligned}
$$

For the equilibrium state of the CRLH transmission line, its meet the conditions:

$L_{R} C_{L}=L_{L} C_{R}$ and $\omega_{0}=\frac{1}{\sqrt[4]{L_{R} C_{R} L_{L} C_{L}}}$ 。

At this point, the simulation results can be used to obtain the transition frequency, and the circuit model of all circuit components parameters are obtained., the parameters of the resulting circuit are compared with the circuit parameters of the initial design stage, and the satisfactory results are obtained. 


\section{Antenna fabrication}

The selected substrate is FR4, relative dielectric constant of FR4 substrate is $\varepsilon_{r}=4.4$, thickness $h=60 \mathrm{mil}$, loss Tan $D=0.025$ 。The cell structure is shown in Figure3.

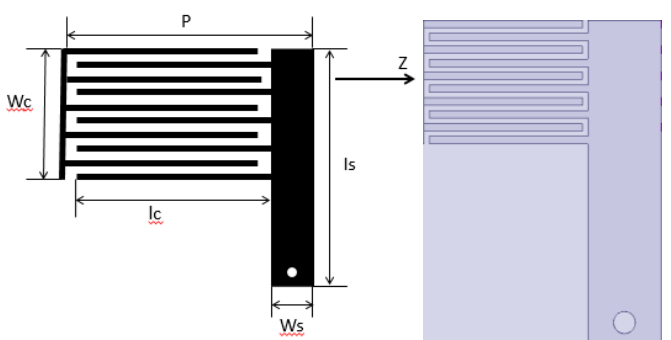

Figure 3 Unit structure diagram of CRLH transmission line type leaky wave antenna

Table1 Parameters of the structural unit of the CRLH transmission line leaky wave antenna

\begin{tabular}{ccccccc} 
& $\mathrm{p}$ & $\mathrm{l}_{\mathrm{c}}$ & $\mathrm{W}_{\mathrm{c}}$ & $\mathrm{l}_{\mathrm{s}}$ & $\mathrm{W}_{\mathrm{s}}$ & $\mathrm{d}$ \\
\hline $\mathrm{FR} 4$ & $4.5 \mathrm{~mm}$ & $4.35 \mathrm{~mm}$ & $3.35 \mathrm{~mm}$ & $8.7 \mathrm{~mm}$ & $2 \mathrm{~mm}$ & $0.3 \mathrm{~mm}$ \\
\hline
\end{tabular}

The parameter $\mathrm{D}$ is the diameter of the earth metal rod. the number of fingers selected for the finger capacitance is 10 , the distance between fingers is $0.15 \mathrm{~mm}$, each finger spacing and edge or stub for $0.15 \mathrm{~mm}$. Antenna physical as shown in Figure4.

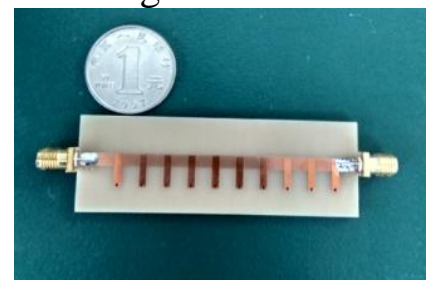

Figure4 Antenna physical

\section{Comparison of simulated and measured results}

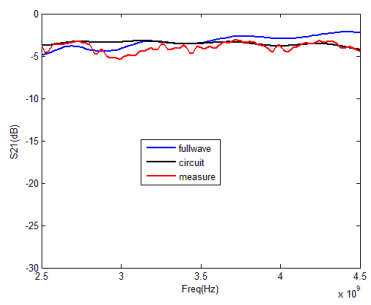

Figure5 Comparison of S parameters(Fullwave,Circuit,Measure)

The results of S parameters are in agreement with the validation of the parameter extraction method.

The comparison of the results of radiation characteristics is given below.

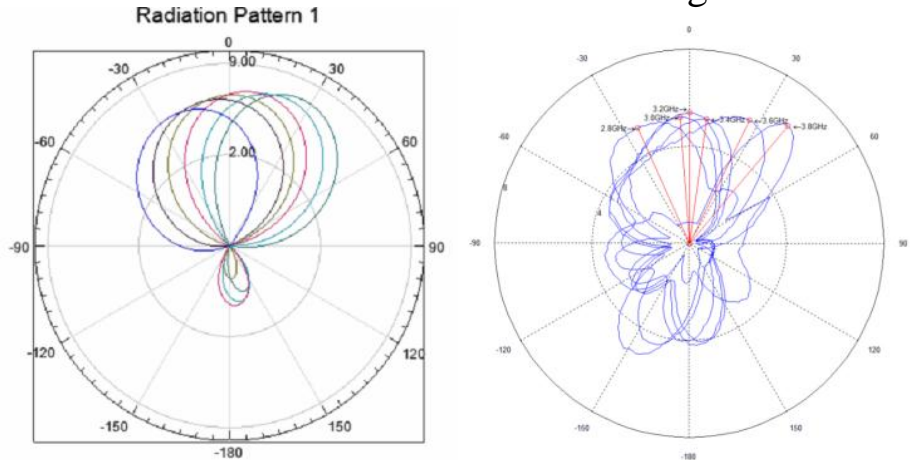

Figure6 The comparison of the results of radiation characteristics 
In order to compare more intuitively, comparing the simulation results of radiation characteristics are given in Table 2 in the US in the antenna and measured results.

Table2 The comparison of the results of radiation characteristics

\begin{tabular}{ccc}
\hline 频率 $(\mathrm{GHz})$ & 定向性 $(\mathrm{dBi})($ 实测 $/$ 仿真) & 波束方向 $\left({ }^{\circ}\right)$ (实测 $/$ 仿真) \\
\hline 2.8 & $5.9225 / 5.7843$ & $-21 /-24$ \\
3 & $6.2921 / 5.7916$ & $-7 /-5$ \\
3.2 & $6.5901 / 5.9707$ & $1 / 0$ \\
3.4 & $6.9418 / 5.7319$ & $10 / 9$ \\
3.6 & $7.1272 / 6.2693$ & $20 / 24$ \\
3.8 & $7.5465 / 7.0152$ & $27 / 38$ \\
\hline
\end{tabular}

\section{Conclusion}

From the measured pattern results and table of data we can see when the frequency increases from $2.8 \mathrm{GHz}$ to $3.8 \mathrm{ghz}$, radiation energy increases slowly. This is because when the frequency increases with the wavelength shorter, increase the electrical size of the antenna, radiation ability also increases.

The antenna designed in this paper has characteristics of both the forward radiation and the back radiation. Perfect realization of the characteristics of the space beam scanning, in each direction of the beam energy is relatively uniform, directed the biggest difference is less than $1.5 \mathrm{dBi}$, and at each frequency point in the beam direction and the simulation results basic consistent.

\section{References}

[1] J.H.Wang, Y. J. Li, Z.Zhang et al. Radiation mechanism and polarization properties of leaky coaxial cables[J]. Radio Science, 2011, vol. 46, No.3.

[2] F.B.Zarrabi, M.Rahimi, Z.Mansouri et al. Miniaturization of microstrip antenna by CRLH-TL technique[J]. Wireless Personal Communications, 2015, 81(3): 1091-1100.

[3] F.B.Zarrabi, R.Ahmadian, M.Rahimi et al. Dual-band antenna designing with CRLH[J]. Microwave and Optical Technology Letters, 2015, 57(4): 774-779.

[4] A.Locatelli, D.Modotto, C.Deangelis, et al. Design of fully printed omnidirectional CRLH loop antennas for WLAN technology[J]. Microwave and Optical Technology Letters, 2014, 56(6): 1405-1408.

[5] K.Saurav, D.Sarkar, K.V.Srivastava. CRLH unit-cell loaded multiband printed dipole antenna[J]. IEEE Antennas and Wireless Propagation Letters, 2014, 13: 852-855. 